100

Statistica Neerlandica (2000) Vol. 54, nr. 1, pp. 100-110

\title{
On a crossroad of resampling plans: bootstrapping elementary symmetric polynomials
}

\author{
A. J. van Es* \\ Korteweg-de Vries Institute for Mathematics, University of Amsterdam, \\ Plantage Muidergracht 24, 1018 TV Amsterdam. The Netherlands \\ R. Helmers** \\ CWI, P.O. Box 94079, 1090 GB Amsterdam, The Netherlands \\ M. Hušková*** \\ Czech Academy of Sciences and Department of Probability and \\ Statistics, Charles University, Sokolovska 83, 18600 Prague, \\ Czech Republic

\begin{abstract}
We investigate the validity of the bootstrap method for the elementary symmetric polynomials $S_{n}^{(k)}=\left(\begin{array}{c}n \\ k\end{array}\right)^{-1} \Sigma_{1 \leq i_{1}<\ldots<i_{k} \leq n} X_{i_{1}} \ldots X_{i_{k}}$ of i.i.d. random variables $X_{1}, \ldots, X_{n}$. For both fixed and increasing order $k$, as $n \rightarrow \infty$ the cases where $\mu=E X_{1} \neq 0$, the nondegenerate case, and where $\mu=\mathrm{E} X_{1}=0$, the degenerate case, are considered.
\end{abstract}

Key Words and Phrases: bootstrap, resampling schemes, U-statistics, nondegenerate polynomials, degenerate polynomials.

\section{Introduction}

In the early days of bootstrap methodology the feeling existed that the bootstrap method which is now usually called the naive nonparametric bootstrap should work in most cases. Nowadays however we know that the range of validity of the naive bootstrap is rather restrictive, typically the standard bootstrap 'only' works when we are dealing with statistics which can be approximated by a sample mean. Hence the standard bootstrap works in any case for asymptotically normal statistics. It also works e.g. for statistics like the Kolmogorov-Smirnov statistic (cf. BICKEL and FreEDMAN, 1981). For other statistics the standard resampling from the empirical distribution has to be replaced by other resampling schemes. To stress the point, some people would say that 'every problem requires its own bootstrap method'.

\footnotetext{
*vanes@wins.uva.nl

**helmers@cwi.nl

*** huskova@karlin.mff.cuni.cz

(C) VVS, 2000. Published by Blackwell Publishers, 108 Cowley Road, Oxford OX4 1JF, UK and 350 Main Street, Malden, MA 02148, USA.
} 
The need for different bootstrap methods can even show up for one statistic. More specific, depending on the asymptotic framework and characteristics of the underlying distribution, it can happen that different resampling methods are required to bootstrap a particular statistic. The statistic we have chosen to investigate is the elementary symmetric polynomial of order $k$.

Let $X_{1}, \ldots, X_{n}$ be independent and identically distributed random variables with common distribution function $F$ and

$$
-\infty<\mu=\mathrm{E} X_{1}<\infty, \quad 0<\sigma^{2}=\sigma^{2}\left(X_{1}\right)<\infty .
$$

Let, for any $1 \leq k \leq n$,

$$
S_{n}^{(k)}=\left(\begin{array}{l}
n \\
k
\end{array}\right)^{-1} \sum_{1 \leq i_{1}<\cdots<i_{k} \leq n} X_{i_{1}} \ldots X_{i_{k}}
$$

and let

$$
F_{n}^{(k)}(x)=P\left(\frac{n^{1 / 2}\left(S_{n}^{(k)}-\mu^{k}\right)}{k \mu^{k-1} \sigma} \leq x\right)
$$

for real $x$. The statistic (1) is called an elementary symmetric polynomial of order $k$. It is frequently used as a typical example of a $\mathrm{U}$-statistic of order $k$.

Let us review some asymptotic results. Asymptotic normality for U-statistics, with a fixed order $k$, has been first derived by HoefFDING (1948). For elementary symmetric polynomials it means that, for $k$ fixed and $\mu \neq 0$, we have

$$
\sup _{x}\left|F_{n}^{(k)}(x)-\Phi(x)\right| \rightarrow 0, \quad \text { as } n \rightarrow \infty,
$$

where $\Phi(x)$ denotes the standard normal distribution function.

For distributions $F$ with $\mu=0$ the $\mathrm{U}$-statistic $S_{n}^{(k)}$ is degenerate since it is readily seen that

$$
\mathrm{E}\left(X_{1} \ldots X_{k} \mid X_{i}\right)=0, \quad i=1, \ldots, n,
$$

whenever $k \geq 2$. The asymptotic distribution of degenerate U-statistics can be found in, e.g. RubiN and Vitale (1980). Note that the limit distributions are no longer normal in the degenerate case (cf. also Theorem 4 of the present paper).

Now consider the standard nonparametric, or naive, bootstrap introduced by EFron (1979). Let $\hat{F}_{n}$ denote the empirical distribution function of the sample $X_{1}, \ldots, X_{n}$ from $F$. Furthermore let $X_{1}^{*}, \ldots, X_{n}^{*}$ denote a bootstrap resample of size $n$, i.e. given the values of $X_{1}, \ldots, X_{n}$ the random variables $X_{1}^{*}, \ldots, X_{n}^{*}$ denote a sample of size $n$ from the empirical distribution $\hat{F}_{n}$. We approximate the distribution $F_{n}^{(k)}(x)$ (c) vvs, 2000

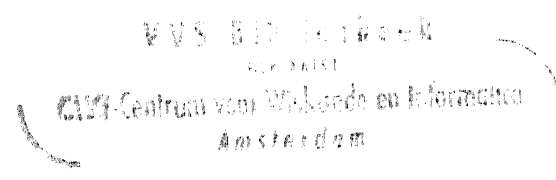


of the normalized $k$ th order elementary symmetric polynomial by its bootstrap counterpart $F_{n}^{(k) *}(x)$, where

$$
F_{n}^{(k)^{*}}(x)=P_{n}^{*}\left(\frac{n^{1 / 2}\left(S_{n}^{(k)^{*}}-\bar{X}_{n}^{k}\right)}{k \bar{X}_{n}^{k-1} s_{n}} \leq x \mid X_{1}, \ldots, X_{n}\right),
$$

with $\bar{X}_{n}$ and $s_{n}$ denoting the sample mean and sample standard deviation of the original sample, and

$$
S_{n}^{(k)^{*}}=\left(\begin{array}{l}
n \\
k
\end{array}\right)^{-1} \sum_{1 \leq i_{1}<\cdots<i_{k} \leq n} X_{i_{1}}^{*} \ldots X_{i_{k}}^{*} .
$$

Here $P_{n}^{*}$ refers to probability under $\hat{F}_{n}$. For $k$ fixed and $\mu \neq 0$, we have bootstrap consistency (cf. Bickel and Freedman, 1981).

$$
\sup _{x}\left|F_{n}^{(k)^{*}}(x)-F_{n}^{(k)}(x)\right| \rightarrow 0, \quad \text { almost surely. }
$$

Similarly, for Studentized elementary symmetric polynomials, one approximates

$$
G_{n}^{(k)}(x)=P\left(\frac{n^{1 / 2}\left(S_{n}^{(k)}-\mu^{k}\right)}{k \bar{X}_{n}^{k-1} S_{n}} \leq x\right)
$$

quite well by

$$
G_{n}^{(k)^{*}}(x)=P_{n}^{*}\left(\frac{n^{1 / 2}\left(S_{n}^{(k)^{*}}-\bar{X}_{n}^{k}\right)}{k\left(\bar{X}_{n}^{*}\right)^{k-1} s_{n}^{*}} \leq x \mid X_{1}, \ldots, X_{n}\right)
$$

where $\bar{X}_{n}^{*}$ and $s_{n}^{*}$ denote the sample mean and sample standard deviation of the bootstrap resample $X_{1}^{*}, \ldots, X_{n}^{*}$. For fixed order Studentized nondegenerate U-statistics Efron's bootstrap has been shown to work better than the classical normal approximation by HeLMERS (1991). It is easy to check that this means that, for $k$ fixed and $\mu \neq 0$, the asymptotic accuracy of the bootstrap approximation $G_{n}^{(k)^{*}}$ to the exact cdf. $G_{n}^{(k)}$ is of order o $\left(n^{-1 / 2}\right)$ a.s., as $n \rightarrow \infty$.

The results above can be summarized by saying that Efron's bootstrap works well in the case of a fixed order nondegenerate elementary symmetric polynomial. In this paper we study two different ways of departing from the standard case. Firstly in Section 2 we investigate the case of increasing order nondegenerate elementary symmetric polynomials. In Section 3 we investigate the case of fixed order degenerate elementary symmetric polynomials. In both cases we show that the bootstrap still works, up to a certain degree and with possible modification of the resampling scheme.

(C) Vvs, 2000 


\section{Nondegenerate polynomials}

Suppose that we are in the case where $\mu$ does not vanish. For this case, the question how far the standard asymptotic normality, stated in (3), still holds if we allow $k$ to increase with $n$, has been investigated in VAN Es and Helmers (1988). It turns out that essentially we have to require $k=\mathrm{o}\left(n^{1 / 2}\right)$ for the polynomials to remain asymptotically normally distributed, with the standardization given by (2). The case where $k \sim \alpha n^{1 / 2}$, for some constant $\alpha>0$ serves as a border case. For results on the asymptotic distribution of the $k$-th root of the polynomials see SzÉKeLY $(1975,1982)$, HaLÁsZ and Székely (1976), Móri and Székely (1982) and VAN Es (1986).

Our first theorem states that Efron's bootstrap still works in cases where the order is allowed to increase, as long as asymptotic normality holds.

THEOREM 1. The bootstrap works, i.e.

$$
\sup _{x}\left|F_{n}^{(k)^{*}}(x)-F_{n}^{(k)}(x)\right| \rightarrow 0,
$$

holds almost surely, with the standard resampling scheme, provided $k=\mathrm{o}\left(n^{1 / 2}\right)$.

The rate of the bootstrap convergence in Theorem 1 is of faster order than $k / \sqrt{n}$, the order of the normal approximation error, as established in VAN Es and HeLMERS (1988). More precisely:

THEOREM 2. If $\mu=\mathrm{E} X_{1} \neq 0,0<\sigma^{2}=\sigma^{2}\left(X_{1}\right), \mathrm{E}\left|X_{1}\right|^{3}<\infty$, and $k=\mathrm{o}\left(n^{1 / 2} \log ^{-1}\right.$ $n \log _{2}^{-1} n$ ), then as both $k$ and $n \rightarrow \infty$,

$$
\frac{n^{1 / 2}}{k} \sup _{x}\left|F_{n}^{(k)}(x)-F_{n}^{(k)^{*}}(x)\right| \rightarrow 0, \quad \text { almost surely, }
$$

where $F_{n}^{(k)}$ denotes the distribution function of $n^{1 / 2}\left(S_{n}^{(k)}-\mu\right) /\left(k \mu^{k-1} \sigma\right)$ and $F_{n}^{(k)^{*}}$ is its bootstrap counterpart (4).

A result, similar to Theorem 2 holds true for Studentized elementary symmetric polynomials. We have the following theorem:

THEOREM 3. If the conditions of Theorem 2 are satisfied and, in addition, $\mathbf{E}\left|X_{1}\right|^{4+\varepsilon}<\infty$, for some $\varepsilon>0$, then as both $k$ and $n \rightarrow \infty$,

$$
\frac{n^{1 / 2}}{k} \sup _{x}\left|G_{n}^{(k)}(x)-G_{n}^{(k)^{*}}(x)\right| \rightarrow 0, \quad \text { almost surely, }
$$

where $G_{n}^{(k)}$ denotes the distribution function of $n^{1 / 2}\left(S_{n}^{(k)}-\mu\right) /\left(k \bar{X}_{n}^{k-1} s_{n}\right)$ and $G_{n}^{(k)^{*}}$ is its bootstrap counterpart (6).

So, the bootstrap approximations $F_{n}^{(k)^{*}}$ respectively $G_{n}^{(k)^{*}}$ are asymptotically closer to $F_{n}^{(k)}$ respectively $G_{n}^{(k)}$ than the normal approximation.

Recent work by PUTTER and VAN ZWET (1998) strongly suggests that the $(4+\varepsilon)$ th moment condition in Theorem 3 can be replaced by the weaker and more natural (C) VvS, 2000 
assumption $\mathrm{E}\left|X_{1}\right|^{3+\varepsilon}<\infty$, for some $\varepsilon>0$. However, we will not pursue this matter here.

EXAMPLE. Consider the special case where the $X_{i}$ are drawn from a discrete distribution concentrated on zero and one. Let $p=P\left(X_{i}=1\right)=1-P\left(X_{i}=0\right)$, with $0<p<1$, and let $E_{n}$ denote the number of ones in the sample. The symmetric polynomial of such $X$ 's can be expressed as the quotient of two binomial coefficients

$$
S_{n}^{(k)}=\left(\begin{array}{c}
n \\
k
\end{array}\right)^{-1}\left(\begin{array}{c}
E_{n} \\
k
\end{array}\right)
$$

If $k=\mathrm{o}\left(n^{1 / 2}\right)$ we have

$$
\frac{n^{1 / 2}}{k}\left(\frac{S_{n}^{(k)}-p^{k}}{p^{k-1} \sqrt{p(1-p)}}\right) \stackrel{\mathcal{D}}{\rightarrow} N,
$$

where $N$ is a standard normal random variable. This confirms Theorem 1 in VAN Es and HeLmers (1988). A detailed proof can be found in VAN Es et al. (1997), an earlier version of this paper. It is readily seen that the naive bootstrap works in this case because the asymptotics are based on a sample average $E_{n} / n$.

Now assume $k \sim \alpha n^{1 / 2}$. Then we have (see VAN Es et al., 1997)

$$
p^{-k} S_{n}^{(k)} \stackrel{\mathcal{D}}{\rightarrow} \mathrm{e}^{-\left(\alpha(p(1-p))^{1 / 2} / p\right) N-\frac{1}{2}\left(\frac{(1-p)}{p}\right) \alpha^{2}}
$$

where $N$ is a standard normal random variable. In this case the naive bootstrap works because, again, the asymptotics are based on a sample average $E_{n} / n$, i.e. with $p_{n}^{*}$ equal to the fraction of ones in the sample, defining

$$
H_{n}^{(k)}(x)=P\left(p^{-k} S_{n}^{(k)} \leq x\right)
$$

and

$$
H_{n}^{(k)^{*}}(x)=P_{n}^{*}\left(\left(p_{n}^{*}\right)^{-k} S_{n}^{(k)^{*}} \leq x\right)
$$

we have uniformly in $x$,

$$
H_{n}^{(k)}(x) \rightarrow H(x) \text { and } H_{n}^{(k)^{*}}(x) \rightarrow H(x), \quad \text { almost surely, }
$$

where $H(x)$ is the distribution function of

$$
\exp \left\{-\frac{\alpha(p(1-p))^{1 / 2}}{p} N-\frac{1-p}{2 p} \alpha^{2}\right\}
$$

C VVS, 2000 
Let us now consider convergence in the norming (2). Note that for this example we have

$$
\begin{aligned}
F_{n}^{(k)}(x) & =P\left(p^{-k} S_{n}^{(k)} \leq \frac{k}{n^{1 / 2}} \frac{(p(1-p))^{1 / 2}}{p} x+1\right) \\
& =H_{n}^{(k)}\left(\frac{k}{n^{1 / 2}} \frac{(p(1-p))^{1 / 2}}{p} x+1\right),
\end{aligned}
$$

and

$$
F_{n}^{(k)^{*}}(x)=H_{n}^{(k)^{*}}\left(\frac{k}{n^{1 / 2}} \frac{\left(p_{n}^{*}\left(1-p_{n}^{*}\right)\right)^{1 / 2}}{p_{n}^{*}} x+1\right)
$$

From (7) it now follows that (5) also holds true, which means that the naive bootstrap also works in this situation.

\section{Degenerate polynomials}

If the order $k$ is fixed then the limit distribution of $S_{n}^{(k)}$ is not normal anymore. It follows from the results of RUBin and VITALE (1980), who investigated the asymptotic distribution of symmetric statistics, that the limit distribution is given by the following theorem:

THEOREM 4. Let $X_{1}, \ldots, X_{n}$ be i.i.d. random variables with zero mean and variance $\sigma^{2}$. Then, as $n \rightarrow \infty$, we have

$$
\sup _{x}\left|P\left(S_{n}^{(k)} \leq x \sigma\left(S_{n}^{(k)}\right)\right)-P\left(\sqrt{k !} H_{k}(Z) \leq x\right)\right| \rightarrow 0
$$

where $H_{k}($.$) is the Hermite polynomial of order k$ and $Z$ is a random variable with a $N(0,1)$ distribution.

The theorem says that $S_{n}^{(k)} / \sigma\left(S_{n}^{(k)}\right)$ has the same limit distribution as

$$
T_{k}=\sum_{*} \frac{\sqrt{k !}}{j ! 2^{(k-j) / 2}\left(\frac{k-j}{2}\right) !} Z^{j}(-1)^{(k-j) / 2}
$$

where $Z$ has a $N(0,1)$ distribution and $\Sigma_{*}$ extends over all indices $0 \leq j \leq k$ such that $(k-j) / 2$ is an integer. It can be checked by straightforward calculations that

$$
\sigma^{2}\left(S_{n}^{(k)}\right)=\sigma^{2 k}\left(\begin{array}{l}
n \\
k
\end{array}\right)^{-1}
$$

Since the form of the distribution is not explicitly known a bootstrap approximation of the distribution of $S_{n}^{(k)}$ is really needed. c vvs, 2000 
It is known that in this case the usual bootstrap does not work for this situation (see BICKEL and FrEEDMAN (1981) for $k=2$ and ARCONES and GINÉ (1992) for general fixed k). The latter authors showed that if the bootstrap version $S_{n}^{(k)^{* *}}$ of the statistic $S_{n}^{(k)}$ is defined as

$$
S_{n}^{(k) * *}=\left(\begin{array}{l}
n \\
k
\end{array}\right)^{-1} \sum_{1 \leq i_{1}<\cdots<i_{k} \leq n}\left(X_{i_{1}}^{*}-\bar{X}_{n}\right) \ldots\left(X_{i_{k}}^{*}-\bar{X}_{n}\right)
$$

the bootstrap works. It says that we should simply copy the original model, where the mean is zero, in the bootstrap world.

Applying the results of ArCones and GrNé (1992) to our situation we get the following result.

THEOREM 5. Let the assumptions of Theorem 4 be satisfied. Then as $n \rightarrow \infty$ we have

$$
\sup _{x}\left|P^{*}\left(S_{n}^{(k) * *} \leq x s_{n}^{k}\left(\begin{array}{l}
n \\
k
\end{array}\right)^{-\frac{1}{2}} \mid X_{1}, \ldots, X_{n}\right)-P\left(\sqrt{k !} H_{k}(Z) \leq x\right)\right| \rightarrow 0,
$$

almost surely, where $s_{n}^{2}$ is the sample variance based on $X_{1}, \ldots, X_{n}$. Hence the ArconesGiné bootstrap is consistent in estimating the distribution of $S_{n}^{(k)} / \sigma\left(S_{n}^{(k)}\right)$.

REMARK. The question is what the limit behavior of $S_{n}^{(k)}$ is, when $k$ increases together with $n$ in such a way that $k=o\left(n^{1 / 2}\right)$, and whether some resampling scheme works in this situation also. Let us assume additionally that $\mathrm{E}\left|X_{i}\right|^{2+\delta}$ is finite for some $\delta>0$. According to the Appendix in RuBIN and Vitale (1980), we have

$S_{n}^{(k)}=\left(\begin{array}{l}n \\ k\end{array}\right)^{-1} \sum_{+} \frac{(-1)^{k-j_{1}-\cdots-j_{k}}}{2^{j_{2}} \ldots k^{j_{k}}} \frac{1}{j_{1} ! \ldots j_{k} !}\left(\sum_{i=1}^{n} X_{i}\right)^{j_{1}}\left(\sum_{i=1}^{n} X_{i}^{2}\right)^{j_{2}} \ldots\left(\sum_{i=1}^{n} X_{i}^{k}\right)^{j_{k}}$,

where $\Sigma_{+}$denotes the summation over $j_{1}, \ldots, j_{k}$ such that $j_{v}=0,1, \ldots, k$ and $\Sigma_{v=1}^{k} v j_{v}=k$. Now, using the Marcinkiewicz-Zygmund strong of large numbers we find that

$$
\frac{1}{n} \sum\left(X_{i}^{2}-1\right) \rightarrow 0, \quad \text { a.s. }
$$

and

$$
n^{-k /(2+\delta)} \sum\left|X_{i}\right|^{k} \rightarrow 0, \quad \text { a.s., } \quad k=3,4, \ldots
$$

(This is valid even for $k \rightarrow \infty$. The reason is the following. Instead of treating $n^{-k /(2+\delta)} \Sigma\left|X_{i}\right|^{k}$ it suffices to treat $n^{-k /(2+\delta)} \Sigma\left|X_{i}\right|^{k} I\left\{\left|X_{i}\right| \leq n^{1 /(2+\delta)}\right\}$ and it can be easily checked that

$$
n^{-k /(2+\delta)} \sum\left|X_{i}\right|^{k} I\left\{\left|X_{i}\right| \leq n^{1 /(2+\delta)}\right\} \leq n^{-k_{0} /(2+\delta)} \sum\left|X_{i}\right|^{k_{0}} I\left\{\left|X_{i}\right| \leq n^{1 /(2+\delta)}\right\}
$$

(C) VVS, 2000 
for any $2+\delta<k_{0} \leq k \leq n$.) Then we carefully treat all terms in (9) to find that only the terms with $j_{1}+2 j_{2}=k$ need not be negligible. All other terms have no influence on the limit distribution. This means that $S_{n}^{(k)}\left(\sigma\left(S_{n}^{(k)}\right)\right)^{-1}$ asymptotically has the same limit distribution as $T_{k}$ (cf. (8)), even for $k \rightarrow \infty$.

Further careful calculations show that the only influential terms are those with $j_{1}+2 j_{2}=k$ and $d_{n 1} \sqrt{k} \leq j_{1} \leq d_{n 2} \sqrt{k}$, where $d_{n 1}$ and $d_{n 2}$ are arbitrary sequences of positive numbers such that $d_{n 1} \rightarrow 0$ and $d_{n 2} \rightarrow \infty$. Concerning the validity of the Arcones-Giné bootstrap under the above-mentioned stronger assumptions, Theorem 5 remains true even for this situation. The reason is that if we replace the arguments based on the strong law of large numbers by the weak law of large numbers arguments everything goes through.

\section{Proofs}

Proof of Theorem 1. We adapt the proof of Theorem 1 in VAN Es and Helmers (1988) to the bootstrap world. The proof is based on the Hoeffding decomposition of elementary symmetric polynomials, as given by KARLIN and RinOTT (1982). For the bootstrap statistic $S_{n}^{(k)^{*}}$ we have

$$
S_{n}^{(k)^{*}}-\bar{X}_{n}^{k}=\sum_{r=1}^{k} H_{r}\left(X_{1}^{*}, \ldots, X_{n}^{*}\right)
$$

where

$$
H_{r}\left(X_{1}^{*}, \ldots, X_{n}^{*}\right)=\left(\begin{array}{c}
n \\
k
\end{array}\right)^{-1}\left(\begin{array}{c}
n-r \\
k-r
\end{array}\right) \bar{X}_{n}^{k-r} \sum_{1 \leq j_{1}<\cdots<j_{r} \leq n} \prod_{i=1}^{r}\left(X_{j_{i}}^{*}-\bar{X}_{n}\right) .
$$

Next define

$$
q_{r}=\frac{\sigma^{2}\left(H_{r+1}\right)}{\sigma^{2}\left(H_{r}\right)}, \quad r=1,2, \ldots, k-1 .
$$

This gives

$$
q_{r}=\frac{s_{n}^{2}}{\bar{X}_{n}^{2}}(k-r)^{2} /((r+1)(n-r)), \quad r=1,2, \ldots, k-1 .
$$

Conditional on $X_{1}, \ldots, X_{n}$, the summands of (10) are uncorrelated. Hence we find, given $X_{1}, \ldots, X_{n}$

$$
\begin{aligned}
\sigma^{2}\left(S_{n}^{(k)^{*}}\right) & =\sum_{r=1}^{k} \sigma^{2}\left(H_{r}\left(X_{1}^{*}, \ldots, X_{n}^{*}\right)\right) \\
& =\sigma^{2}\left(H_{1}\left(X_{1}^{*}, \ldots, X_{n}^{*}\right)\right)\left(1+q_{1}+q_{1} q_{2}+\cdots+q_{1} q_{2} \ldots q_{k-1}\right) .
\end{aligned}
$$

(c) VVS, 2000 
Since on a set of probability one we have $s_{n} \rightarrow \sigma$ and $\bar{X}_{n} \rightarrow \mu$, by the assumption $k=\mathrm{o}\left(n^{1 / 2}\right)$, with probability one we have, for fixed $k$ and $n$ and for $n$ sufficiently large,

$$
q_{r}<c \frac{k^{2}}{r n}, \quad r=1,2, \ldots, k-1
$$

for some constant $c>0$. This implies

$$
0 \leq \sum_{r=2}^{k} q_{1} \ldots q_{r-1} \leq \sum_{r=2}^{\infty} \frac{1}{(r-1) !}\left(\frac{c k^{2}}{n}\right)^{r-1}=\mathrm{e}^{c\left(k^{2} / n\right)}-1=\mathrm{o}(1),
$$

almost surely, as $n \rightarrow \infty$, which shows that the linear term

$$
H_{1}\left(X_{1}^{*}, \ldots, X_{n}^{*}\right)=k n^{-1} \bar{X}_{n}^{k-1} \sum_{i=1}^{n}\left(X_{i}^{*}-\bar{X}_{n}\right)
$$

is the dominant term in the expansion (10). The result now follows from the central limit theorem for triangular arrays.

Proof of Theorem 2. It is proved in the appendix of VAN Es and Helmers (1988) that

$$
\begin{aligned}
F_{n}^{(k)}(x)= & \Phi(x)+\frac{1}{6} n^{-1 / 2} \phi(x)\left(1-x^{2}\right)\left\{\sigma^{-3} \mathrm{E}\left(X_{1}-\mu\right)^{3}+3(k-1) \sigma \mu^{-1}\right\} \\
& +o\left(\frac{k}{n^{1 / 2}}\right)
\end{aligned}
$$

uniformly in all real $x$. Here $\phi$ of course denotes the standard normal density. Note that there is no need for the usual requirement that $F$ is non-lattice, when $k \rightarrow \infty$, as $n \rightarrow \infty$. (However, if $k$ is fixed, we must of course add the assumption that $F$ is nonlattice, in order to guarantee that our expansion is valid uniformly.) It is now easy to check that the argument leading to the expansion for $F_{n}^{(k)}$ can be repeated to find that, quite similarly, also

$$
F_{n}^{(k)^{*}}(x)=\Phi(x)+\frac{1}{6} n^{-1 / 2} \phi(x)\left(1-x^{2}\right)\left\{s_{n}^{-3} m_{3}+3(k-1) s_{n} \bar{X}_{n}^{-1}\right\}+o\left(\frac{k}{n^{1 / 2}}\right)
$$

holds true almost surely. Here $m_{3}$ of course denotes the sample third central moment $n^{-1} \sum_{i=1}^{n}\left(X_{i}-\bar{X}_{n}\right)^{3}$ of the original sample. Comparing (11) with (12) we easily conclude that, because almost surely $\bar{X}_{n} \rightarrow \mu, s_{n}^{2} \rightarrow \sigma^{2}, m_{3} \rightarrow \mathrm{E}\left(X_{1}-\mu\right)^{3}$ by the strong law, the theorem is proved.

(c) Vvs, 2000 
Proof OF THeOREM 3. We only give an outline of the method of proof and omit further messy details. It can be proved by a slight adaptation of the proof given in MAESONO (1995) (cf. also Helmers (1991) and VAN Es and Helmers (1988)) that

$$
\begin{aligned}
G_{n}^{(k)}(x)= & \Phi(x)+\frac{1}{6} n^{-1 / 2} \phi(x) \\
& \times\left\{\left(2 x^{2}+1\right) \sigma^{-3} \mathrm{E}\left(X_{1}-\mu\right)^{3}+3(k-1)\left(x^{2}+1\right) \sigma \mu^{-1}\right\}+o\left(\frac{k}{n^{1 / 2}}\right)
\end{aligned}
$$

uniformly in all real $x$. The main new ingredient in the present proof is to verify that the Studentization we employ - which simply amounts to replacing the scaling factor $k \mu^{k-1} \sigma$ by the plug-in estimate $k \bar{X}_{n}^{k-1} s_{n}$ - will yield exactly the same Edgeworth expansion (cf. (13)) as Studentization by means of the delete-one-jackknife method, which is applied in Helmers (1991) and Maesono (1995). Combination of this fact with an argument like the one described in the appendix of VAN Es and HeLMERS (1988) will then complete our proof. Similarly, one can also show that

$$
G_{n}^{(k)^{*}}(x)=\Phi(x)+\frac{1}{6} n^{-1 / 2} \phi(x)\left\{\left(2 x^{2}+1\right) s_{n}^{-3} m_{3}+3(k-1)\left(x^{2}+1\right) s_{n} \bar{X}_{n}^{-1}\right\}+o\left(\frac{k}{n^{1 / 2}}\right)
$$

holds true almost surely. Comparing (13) with (14), we easily conclude that, because almost surely $\bar{X}_{n} \rightarrow \mu, s_{n}^{2} \rightarrow \sigma^{2}, m_{3} \rightarrow \mathrm{E}\left(X_{1}-\mu\right)^{3}$ by the strong law, the theorem is proved.

\section{Acknowledgements}

The authors are grateful to two referees for their helpful reviews. The research by M. Hušková was partially supported by grant GAČR 201/97/1163 of Charles University Prague, and the focus area 'Computationally Intensive Methods in Stochastics' of the Netherlands Organization for Scientific Research (NWO).

\section{References}

Arcones, M. A. and E. GrNE (1992), On the bootstrap of U and V statistics, The Annals of Statistics 20, 655-674.

Bickel, P. and D. FReEDMAN (1981), Some asymptotic theory for the bootstrap, The Annals of Statistics 9, 1196-1217.

Efron, B. (1997), Bootstrap methods: another look at the jackknife, The Annals of Statistics 11, $1-27$.

VAN Es, A. J. (1986), On the weak limits of elementary symmetric polynomials, The Annals of Probability 14, 677-695.

VAN Es, A. J. and R. Helmers (1988), Elementary symmetric polynomials of increasing order, Probability Theory and their Related Fields 80, 21-35.

VAN Es, A. J., R. Helmers and M. HuŠKovÁ (1997), On a crossroad of resampling plans: bootstrapping elementary symmetric polynomials, Technical Report PNA-R9704, Centrum voor Wiskunde en Informatica, Amsterdam.

(c) VVS, 2000 
HalÁsz, G. and G. J. SzÉkely (1976), On the elementary symmetric polynomials of independent random variables, Acta Mathematica Academiae Scientiarum Hungaricae 28, 397-400.

Helmers, R. (1991), On the Edgeworth expansion and the bootstrap approximation for a studentized U-statistic, The Annals of Statistics 19, 470-484.

HoefFDING, W. (1948), A class of statistics with asymptotically normal distribution, The Annals of Mathematical Statistics 19, 293-325.

KARLIN, S. and Y. RINOTT (1982), Applications of ANOVA type decompositions for conditional variance statistics including jackknife estimates, The Annals of Statistics 10, 485-501.

Maesono, Y. (1995), On the normal approximations of Studentized U-Statistic, Journal of the Japanese Statistical Society 25, 19-33.

Móri, T. F. and G. J. Székely (1982), Asymptotic behaviour of symmetric polynomial statistics, The Annals of Probability 10, 124-131.

PUTTER, H. and W. R. VAN ZWET (1998), Empirical Edgeworth expansions for symmetric statistics, The Annals of Statistics 26, 1540-1569.

Rubin, H. and R. A. Vitale (1980), Asymptotic distribution of symmetric statistics, The Annals of Statistics 8, 165-170.

SzÉKELY, G. J. (1975), On the polynomials of independent random variables, in: P. RÉvész, Limit theorems of probability theory, Colloquia Mathematica Societatis, Janos Bolyai, North Holland, 365-371.

SZÉKELY, G. J. (1982), A limit theorem for elementary symmetric polynomials of independent random variables, Zeitschrift für Wahrscheinlichkeitstheorie und Verwandte Gebiede $\mathbf{5 9}$ $355-359$.

Received: February 1997. Revised February 1999. 